\title{
Nucleus-mediated spin-flip transitions in GaAs quantum dots
}

\author{
Sigurdur I. Erlingsson, ${ }^{1}$ Yuli V. Nazarov, ${ }^{1}$ and Vladimir I. Fal'ko ${ }^{2}$ \\ ${ }^{1}$ Delft University of Technology, Department of Applied Physics, Lorentzweg 1, 2628 Delft, The Netherlands \\ ${ }^{2}$ School of Physics and Chemistry, Lancaster University, Lancaster, LA1 4YB, United Kingdom \\ (Received 9 April 2001; revised manuscript received 18 July 2001; published 12 October 2001)
}

\begin{abstract}
Spin-flip rates in GaAs quantum dots can be quite slow, thus opening up the possibilities to manipulate spin states in the dots. We present here estimations of inelastic spin-flip rates mediated by hyperfine interaction with nuclei. Under general assumptions the nucleus-mediated rate is proportional to the phonon relaxation rate for the corresponding non-spin-flip transitions. The rate can be accelerated in the vicinity of a singlet-triplet excited state crossing. The small proportionality coefficient depends inversely on the number of nuclei in the quantum dot. We compare our results with known mechanisms of spin-flip in GaAs quantum dots.
\end{abstract}

DOI: 10.1103/PhysRevB.64.195306

PACS number(s): 73.21.La

\section{INTRODUCTION}

The electron spin states in bulk semiconductor and heterostructures have attracted much attention in recent years. Experiments indicate very long spin decoherence times and small transition rates between states of different spin. ${ }^{1-3}$ These promising results have motivated proposals for information processing based on electron spins in quantum dots, which might lead to a realization of a quantum computer.,

A quantum dot is a region where electrons are confined. The energy spectrum is discrete, due to the small size, and can display atomiclike properties. ${ }^{6,7}$ Here we will consider quantum dots in GaAs-AlGaAs heterostructures. The main reasons for studying them are that relevant quantum dots are fabricated in such structures and GaAs has peculiar electron and phonon properties which are of interest. There are two main types of gate controlled dots in these systems, so-called vertical and lateral dots. ${ }^{8}$ They are characterized by different transverse confinement, which is approximately a triangular well and a square well for the lateral and vertical dots, respectively.

Manipulation of the electron spin in a coherent way requires that it should be relatively well isolated from the surrounding environment. Coupling a quantum dot, or any closed quantum system, to its environment can cause decoherence and dissipation. One measure of the strength of the coupling to the environment is the transition rates, or inverse lifetimes, of the quantum dot states. Calculations of transition rates between different spin states due to phononassisted spin-flip process mediated by spin-orbit coupling, which is one possibility for spin relaxation, have given surprisingly low rates in quantum dots. ${ }^{9-11}$ For these calculations it is very important that the electron states are discrete, and the result differs strongly from that obtained in application to two-dimensional (2D) extended electron states in GaAs. The same argument applies to the phonon-scattering mechanisms, since certain phonon processes possible in $2 \mathrm{D}$ and $3 \mathrm{D}$ electron systems are not effective in scattering the electron in $0 \mathrm{D}$. An alternative mechanism of spin relaxation in quantum dots is caused by hyperfine coupling of nuclear spins to those of electrons. Although the hyperfine interaction mediated spin relaxation in donors was considered a long time ago, ${ }^{12}$ no analysis has been made yet, to our knowledge, of the hyperfine interaction mediated spin-flip processes in quantum dots.

The present paper offers an estimation of the scale of hyperfine interaction induced spin relaxation rates in GaAs quantum dots and its magnetic-field dependence, the main result presented by the expression in Eq. (12). Since the parameters of hyperfine interaction between conduction-band electrons and underlying nuclei in GaAs have been extensively investigated, ${ }^{13,14}$ including the Overhauser effect and spin relaxation in GaAs/AlGaAs heterostructures, ${ }^{15-19}$ we are able now to predict the typical time scale for this process, in particular quantum dot geometries. The rate that we find depends inversely on the number of nuclei in the dot (which can be manipulated by changing the gate voltage) and is proportional to the inverse squared exchange splitting in the dot (which can be varied by application of an external magnetic field with orientation within the 2D plane). The following text is organized in two sections: Sec. II, where the transition rates in systems with discrete spectra are analyzed, and Sec. III, where the obtained result is compared to transition rates provided by the spin-orbit coupling mechanism.

\section{MODEL AND ASSUMPTIONS}

The ground state of a quantum dot is a many-electron singlet $\left|S_{g}\right\rangle$, for sufficiently low magnetic fields. This can change at higher magnetic fields. We assume that the system is in a regime of magnetic field so that the lowest-lying states are ordered as shown in Fig. 1. The relevant energy scales used in the following analysis are given by the energy difference between the triplet state (we assume small Zeeman splitting) and the ground state $\varepsilon=E_{T^{\prime}}-E_{g}$, and exchange splitting $\delta_{S T}=E_{S^{\prime}}-E_{T^{\prime}}$ between the first excited singlet and the triplet. It is possible to inject an electron into an excited state of the dot. If this excited state is a triplet, the system may get stuck there since a spin flip is required to cause transitions to the ground state.

The $\Gamma$ point of the conduction band in GaAs is mainly composed of $s$ orbitals, so that the hyperfine interaction can be described by the contact interaction Hamiltonian ${ }^{20}$

$$
H_{\mathrm{HF}}=A \sum_{i, k} \boldsymbol{S}_{i} \cdot \boldsymbol{I}_{k} \delta\left(\boldsymbol{r}_{i}-\boldsymbol{R}_{k}\right),
$$




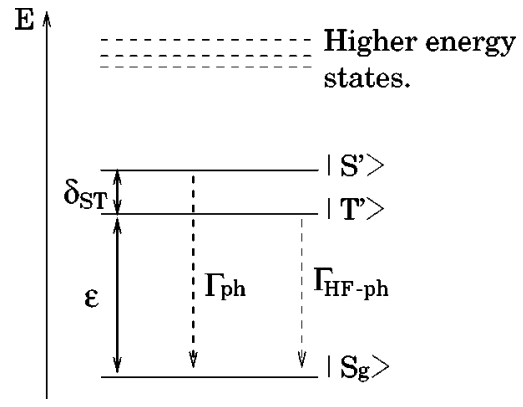

FIG. 1. The lowest lying states of the quantum dot. The energy separation of the ground-state singlet and the first triplet is denoted by $\varepsilon$ and the exchange splitting by $\delta_{S T}$. The two rates indicated are the phonon rate $\Gamma_{\mathrm{ph}}$ and the combined hyperfine and phonon rate $\Gamma_{\text {HF-ph }}$

where $\boldsymbol{S}_{i}\left(\boldsymbol{I}_{k}\right)$ and $\boldsymbol{r}_{i}\left(\boldsymbol{R}_{k}\right)$ denote the spin and position the $i$ th electron ( $k$ th nuclei). This coupling flips the electron spin and simultaneously lowers/raises the $z$ component of a nuclear spin, which mixes spin states and provides the possibility for relaxation.

But the hyperfine interaction alone does not guarantee that transitions between the above-described states occur, since the nuclear spin-flip cannot relax the excessive initial-state energy. (The energy associated with a nuclear spin is the nuclear Zeeman, $\hbar \omega_{n}$, energy which is three orders of magnitude smaller than the electron Zeeman energy and the energies related to the orbital degree of freedom.) For free electrons, the change in energy accompanying a spin-flip caused by the hyperfine scattering is compensated by an appropriate change in its kinetic energy. In the case of a quantum dot, or any system with a discrete energy spectrum, this mechanism is not available and no hyperfine induced transitions will occur because the energy released by the quantum dot cannot be absorbed. Therefore the spin-relaxation process in a dot also requires taking into account the electron coupling to the lattice vibrations. The excess energy from the quantum dot can be emitted in the form of a phonon. Since the "bare" electron-phonon interaction $H_{\mathrm{ph}}$ does not contain any spin operators and thus does not couple directly different spin states, one has to employ second-order perturbation theory which results in transitions via virtual states. The amplitude of such a transition between the triplet state $\left|T^{\prime}\right\rangle$ and the ground state $\left|S_{g}\right\rangle$ is

$$
\begin{aligned}
\left\langle T^{\prime} \mid S_{g}\right\rangle= & \sum_{t} \frac{\left\langle T^{\prime}\left|H_{\mathrm{ph}}\right| t\right\rangle\left\langle t\left|H_{\mathrm{HF}}\right| S_{g}\right\rangle}{E_{T^{\prime}}-\left(E_{t}+\hbar \omega_{q}\right)} \\
& +\sum_{s} \frac{\left\langle T^{\prime}\left|H_{\mathrm{HF}}\right| s\right\rangle\left\langle s\left|H_{\mathrm{ph}}\right| S_{g}\right\rangle}{E_{T^{\prime}}-\left(E_{s}+\hbar \omega_{n}\right)}
\end{aligned}
$$

where $\hbar \omega_{q}$ is the energy of the emitted phonon and $\hbar \omega_{n}$ $\approx 0$ is the energy changed by raising/lowering a nuclear spin.

It is natural to assume that the exchange splitting is smaller than the single-particle level splitting, so that the dominating contribution to the amplitude $\left\langle T^{\prime} \mid S_{g}\right\rangle$ comes from the term describing the virtual state $|s\rangle=\left|S^{\prime}\right\rangle$, due to a small denominator. All other terms can be ignored and the approximate amplitude takes the form

$$
\left\langle T^{\prime} \mid S_{g}\right\rangle \approx \frac{\left\langle T^{\prime}\left|H_{\mathrm{HF}}\right| S^{\prime}\right\rangle\left\langle S^{\prime}\left|H_{\mathrm{ph}}\right| S_{g}\right\rangle}{E_{T^{\prime}}-E_{S^{\prime}}} .
$$

The justification for this assumption is that we aim at obtaining estimates of the rates, and including higher states would not affect the order of magnitude, even if the exchange splitting is substantial. Note that the phonon and nuclear state are not explicitly written in Eq. (3).

The transition rate from $\left|T^{\prime}\right\rangle$ to $\left|S_{g}\right\rangle$ is given by Fermi's golden rule,

$$
\widetilde{\Gamma}_{\mathrm{HF}-\mathrm{ph}}=\frac{2 \pi}{\hbar} \sum_{N_{q}^{\prime}, \boldsymbol{\mu}^{\prime}}\left|\left\langle T^{\prime} \mid S_{g}\right\rangle\right|^{2} \delta\left(E_{\mathrm{i}}-E_{\mathrm{f}}\right),
$$

where $N_{\boldsymbol{q}}^{\prime}$ and $\boldsymbol{\mu}^{\prime}$ are the final phonon and nuclear states, respectively, and $E_{\mathrm{i}}$ and $E_{\mathrm{f}}$ stand for the initial and final energies. Inserting Eq. (3) into Eq. (4) and averaging over initial nuclear states with probability $P(\boldsymbol{\mu})$, we obtain an approximate equation for the nucleus mediated transition rate,

$$
\begin{aligned}
\Gamma_{\mathrm{HF}-\mathrm{ph}}= & \frac{2 \pi}{\hbar} \sum_{N_{\boldsymbol{q}}^{\prime}}\left|\left\langle S^{\prime} ; N_{\boldsymbol{q}}^{\prime}\left|H_{\mathrm{ph}}\right| S_{g} ; N_{\boldsymbol{q}}\right\rangle\right|^{2} \delta\left(E_{\mathrm{i}}-E_{\mathrm{f}}\right) \\
& \times \sum_{\boldsymbol{\mu}^{\prime}, \boldsymbol{\mu}} \frac{P(\boldsymbol{\mu})\left|\left\langle T^{\prime} ; \boldsymbol{\mu}^{\prime}\left|H_{\mathrm{HF}}\right| S^{\prime} ; \boldsymbol{\mu}\right\rangle\right|^{2}}{\left(E_{T^{\prime}}-E_{S^{\prime}}\right)^{2}} \\
= & \Gamma_{\mathrm{ph}}(\varepsilon) \sum_{\boldsymbol{\mu}^{\prime}, \boldsymbol{\mu}} \frac{P(\boldsymbol{\mu})\left|\left\langle T^{\prime} ; \boldsymbol{\mu}^{\prime}\left|H_{\mathrm{HF}}\right| S^{\prime} ; \boldsymbol{\mu}\right\rangle\right|^{2}}{\left(E_{T^{\prime}}-E_{S^{\prime}}\right)^{2}}
\end{aligned}
$$

where $\Gamma_{\mathrm{ph}}$ is the non-spin-flip phonon rate as a function of the relaxed energy $\varepsilon=E_{T^{\prime}}-E_{S_{g}}$.

We will approximate the many-body orbital wave functions by symmetric, $\left|\Psi_{S}\right\rangle$, and antisymmetric, $\left|\Psi_{T}\right\rangle$, Slater determinants corresponding to the singlet and triplet states, respectively. It is not obvious a priori why this approximation is applicable, since the Coulomb interaction in few electron quantum dots can be quite strong. ${ }^{21,22}$ The exact energy levels are very different from those obtained by simply adding the single-particle energies. However, the wave function will not drastically change and especially the matrix elements calculated using Slater determinants are comparable to the ones obtained by using exact ones. The singlet and triplet wave functions can be decomposed into orbital and spin parts: $\left|T^{\prime}\right\rangle=\left|\Psi_{T}\right\rangle|T\rangle$ and $|S\rangle=\left|\Psi_{S}\right\rangle|S\rangle$, where

$$
|T\rangle=-\frac{\nu_{x}-i \nu_{y}}{\sqrt{2}}|1,+1\rangle+\frac{\nu_{x}+i \nu_{y}}{\sqrt{2}}|1,-1\rangle+\nu_{z}|1,0\rangle \text {. }
$$

Using the above discussed wave functions in Eq. (6) we obtain the following: 


$$
\begin{aligned}
\sum_{\boldsymbol{\mu}^{\prime} \boldsymbol{\mu}} & P(\boldsymbol{\mu})\left|\left\langle T^{\prime} ; \boldsymbol{\mu}\left|H_{\mathrm{HF}}\right| S^{\prime} ; \boldsymbol{\mu}^{\prime}\right\rangle\right|^{2} \\
& =\frac{A^{2}}{4} G_{\mathrm{corr}} \sum_{k}\left(\left|\Psi_{1}\left(\boldsymbol{R}_{k}\right)\right|^{2}-\left|\Psi_{2}\left(\boldsymbol{R}_{k}\right)\right|^{2}\right)^{2},
\end{aligned}
$$

where $\Psi_{1,2}$ are the wave functions of the lowest energy states. The factor $G_{\text {corr }}$ contains the nuclear correlation functions

$$
G_{\mathrm{corr}}=\sum_{\eta, \gamma=x, y, z} \nu_{\eta} \nu_{\gamma}^{*}\left(\bar{G}_{\eta \gamma}+\frac{1}{2} i \sum_{\kappa} \epsilon_{\gamma \eta \kappa}\left\langle I_{\kappa}\right\rangle\right) .
$$

Here we have introduced symmetric part of the nuclear correlation tensor $\bar{G}_{\eta \gamma}=\left\langle\delta I_{\eta} \delta I_{\gamma}+\delta I_{\gamma} \delta I_{\eta}\right\rangle / 2$, where $\delta I_{\eta}=I_{\eta}$ $-\left\langle I_{\eta}\right\rangle$. The $\nu_{\eta}$ 's are the coefficients in the triplet state expansion in Eq. (7) and $\epsilon_{\gamma \eta \kappa}$ is the totally antisymmetric tensor. We assume that nuclei are identical and noninteracting (thus we can drop the $k$ subscript), which gives for an isotropic system $G_{\text {corr }}=I(I+1) / 3=1.25$ since Ga and As both have nuclear spin $I=3 / 2$.

Let us now introduce the length scales $l$ and $z_{0}$ which are the spatial extent of the electron wave function in the lateral direction and the dot thickness, respectively. Let $C_{n}$ denote concentration of nuclei with nonzero spin. The effective number of nuclei contained within the quantum dot is

$$
N_{\text {eff }}=C_{n} l^{2} z_{0} .
$$

In GaAs $N_{\text {eff }} \gg 1$ and the sum over the nuclei in Eq. (8) can be replaced by $C_{n} \int d^{3} \boldsymbol{R}_{k}$ and we define the dimensionless quantity

$$
\gamma_{\text {int }}=l^{2} z_{0} \int d^{3} \boldsymbol{R}_{k}\left[\left|\Psi_{1}\left(\boldsymbol{R}_{k}\right)\right|^{2}-\left|\Psi_{2}\left(\boldsymbol{R}_{k}\right)\right|^{2}\right]^{2} .
$$

To relate the hyperfine constant $A$ (which has dimension energy $\times$ volume) to a more convenient parameter we note that the splitting of spin-up and spin-down states at maximum nuclear polarization is $E_{n}=A C_{n} I$, where $I=3 / 2$ is the nuclear spin. Thus the hyperfine mediated transition rate is

$$
\Gamma_{\mathrm{HF}-\mathrm{ph}}=\Gamma_{\mathrm{ph}}(\varepsilon)\left(\frac{E_{n}}{\delta_{S T}}\right)^{2} \frac{G_{\mathrm{corr}} \gamma_{\mathrm{int}}}{(2 I)^{2} N_{\mathrm{eff}}} .
$$

Note that the rate is inversely proportional to the number of nuclei $N_{\text {eff }}$ in the quantum dot and depends on the inverse square of $\delta_{S T}$, which are both possible to vary in experiments. $^{23,25}$ In particular, the singlet-triplet splitting of excited states of a dot can be brought down to zero value using magnetic field parallel to the $2 \mathrm{D}$ plane of the heterostructure, which would accelerate the relaxation process. The nuclear correlation functions in $G_{\text {corr }}$ may also be manipulated by optical orientation of the nuclear system. ${ }^{13,14}$

\section{COMPARISON AND ESTIMATES}

We now consider Eq. (12) for a specific quantum dot structure. It is assumed that the lateral confinement is parabolic and that the total potential can be split into a lateral and transverse part. For vertical dots the approximate transverse wave function is

$$
\chi^{\operatorname{ver}}(z)=\left(\frac{2}{z_{0}}\right)^{1 / 2} \sin \left(\frac{\pi z}{z_{0}}\right)
$$

where $z_{0}$ is the thickness of the quantum well, i.e., the dot thickness. The wave functions in the lateral direction are the Darwin-Fock solutions $\phi_{n, l}(x, y)$ with radial quantum number $n$ and angular momentum $l$. The single-particle states corresponding to $(n, l)=(0,0)$ and $(0, \pm 1)$ are used to construct the Slater determinant for a two-electron quantum dot. In the case of these states the factor $\gamma_{\text {int }}$ in Eq. (11) is then $\gamma_{\text {int }}=0.12$ for a vertical dot and $\gamma_{\text {int }}=0.045$ for a lateral one.

One property of the Darwin-Fock solution is the relation $l^{-2}=\hbar \omega m^{*} / \hbar^{2}$ where $\omega=\sqrt{\Omega_{0}^{2}+\omega_{c}^{2} / 4}$ is the effective confining frequency and $\omega_{c}=\mathrm{eB} / \mathrm{m}^{*}$ the cyclotron frequency. Inserting this and Eq. (10) into Eq. (12) the rate for parabolic quantum dots becomes

$$
\Gamma_{\mathrm{HF}-\mathrm{ph}}=\Gamma_{\mathrm{ph}}(\varepsilon) \hbar \omega\left(\frac{E_{n}}{\delta_{S T}}\right)^{2} \frac{G_{\mathrm{corr}} \gamma_{\mathrm{int}}}{(2 I)^{2}}\left(\frac{m^{*}}{\hbar^{2} C_{n} z_{0}}\right) .
$$

Spin relaxation due to spin-orbit related mechanisms in GaAs quantum dots were investigated by Khaetskii and Nazarov in Refs. 10 and 9. We will summarize their results here for comparison with our hyperfine-phonon mechanism. In Ref. 10, it has been found that the dominating scattering mechanism is due to the absence of inversion symmetry. There are three rates related to this mechanism,

$$
\begin{gathered}
\Gamma_{1}=\Gamma_{\mathrm{ph}}(\varepsilon) \frac{8}{3}\left(\frac{m^{*} \beta^{2}}{\hbar \omega}\right)^{3}, \\
\Gamma_{2}=\Gamma_{\mathrm{ph}}(\varepsilon) \frac{7}{24} \frac{\left(m^{*} \beta^{2}\right)(\hbar \omega)}{E_{z}^{2}} \\
\Gamma_{5}=\Gamma_{\mathrm{ph}}(\varepsilon) 6\left(m^{*} \beta^{2}\right)\left(g^{*} \mu_{\mathrm{B}} B\right)^{2} \frac{\hbar \omega}{\left(\hbar \Omega_{0}\right)^{4}} .
\end{gathered}
$$

The Hamiltonian representing the absence of inversion symmetry has two distinct contribution which behave differenty under a certain unitary transformation. ${ }^{10}$ This behavior results in the two different rates in Eqs. (15) and (16). The inclusion of Zeeman splitting gives the rate in Eq. (17). Here, $m^{*} \beta^{2}$ is determined by the transverse confinement and band structure parameters and $E_{z}=\left\langle p_{z}^{2}\right\rangle /\left(2 m^{*}\right)$. For vertical dots of thickness $z_{0}=15 \mathrm{~nm}$, then $m^{*} \beta^{2} \approx 4 \times 10^{-3} \mathrm{meV}$, but one should be cautious when considering a different thicknesses, since $m^{*} \beta^{2} \propto z_{0}^{-4}$, so the rates are sensitive to variations in $z_{0}$. The transition rates in Eqs. (12), (15), and (16) are all proportional to the phonon rate $\Gamma_{\mathrm{ph}}$ evaluated for the same energy difference $\varepsilon$. It is thus sufficient to compare the only the proportionality coefficients.

Let us now consider for which confining energies the different rates are comparable. At zero magnetic field $\Gamma_{1}=\Gamma_{2}$ at $\hbar \Omega_{0} \approx 0.8 \mathrm{meV}$. The estimated confining energies of vertical quantum dots used in experiment are in the range 
2-5.5 meV. ${ }^{7,24,25}$ For those dots $\Gamma_{2} \gg \Gamma_{1}$ due to the very different dependence on the confinement, $\Gamma_{1} \propto\left(\hbar \Omega_{0}\right)^{-3}$ and $\Gamma_{2} \propto \hbar \Omega_{0}$. Doing the same for $\Gamma_{1}$ and $\Gamma_{\text {HF-ph }}$ we obtain that those rates are equal at $\hbar \Omega_{0} \approx 4.4 \mathrm{meV}$. The numerical values used for the hyperfine rate in Eq. (12) are the following: $E_{n}=0.13 \mathrm{meV},{ }^{15} \delta_{S T}=2.3 \mathrm{meV},{ }^{26}$ and a $z_{0}=15 \mathrm{~nm}$. Thus for $B=0 \mathrm{~T}$ and the previously cited experimental values for the confining energy the dominant transition rate is Eq. (16).

For clarity we will give the values of the rates. The nonspin-flip rate $\Gamma_{\mathrm{ph}}(\varepsilon)$ is given in Ref. 10 and using the values $\hbar \Omega_{0}=5.5 \mathrm{meV}$ and $B=0 \mathrm{~T}$ we get

$$
\begin{gathered}
\Gamma_{\mathrm{ph}} \approx 3.6 \times 10^{7} \mathrm{~s}^{-1}, \\
\Gamma_{\mathrm{HF}-\mathrm{ph}} \approx 2 \times 10^{-2} \mathrm{~s}^{-1}, \\
\Gamma_{2} \approx 1 \times 10^{2} \mathrm{~s}^{-1} .
\end{gathered}
$$

The values of level separations $\varepsilon=2.7 \mathrm{meV}$ and $\delta_{S T}$ $=2.84 \mathrm{meV}$ are taken from Ref. 25 .

An application of a magnetic field to the dot may result in two effects. The rate $\Gamma_{5}$ becomes larger than $\Gamma_{2}$ for magnetic fields around $B=1 \mathrm{~T}\left(\hbar \Omega_{0}=3 \mathrm{meV}\right)$ to $B \approx 5.4 \mathrm{~T}\left(\hbar \Omega_{0}\right.$ $=5.5 \mathrm{meV}$ ). More importantly, the exchange splitting $\delta_{S T}$ can vanish in some cases and the hyperfine rate in Eq. (12) will dominate. It is also worth noting that in this limit the approximation used in obtaining Eq. (3) becomes very good. Since the rates considered here are all linear in the phonon rate the divergence of $\Gamma_{\mathrm{ph}}$ at the singlet-triplet transition does not affect the ratio of the rates. The above estimates were focused on vertical dots. To obtain the corresponding results for lateral dots the value of $\gamma_{\text {int }}$ in Eq. (14) should be used.

In summary, we have calculated the nucleus mediated spin-flip transition rate in GaAs quantum dots. The comparison of our results to those previously obtained for the spinorbit scattering mechanism indicates that the rates we obtained here are relatively low, due to the discrete spectrum, so we believe that hyperfine interaction would not cause problems for spin-coherent manipulation with GaAs quantum dots. Nevertheless, the hyperfine rate, which was found to be lower than the spin-orbit rates at small magnetic field, may diverge and become dominant at certain values of magnetic field corresponding to the resonance between triplet and singlet excited states in the dot.

\section{ACKNOWLEDGMENTS}

This work is a part of the research program of the "Stichting vor Fundementeel Onderzoek der Materie (FOM)," EPSRC, and INTAS. One of the authors (V.F.) acknowledges support from NATO CLG and thanks R. Haug and I. Aleiner for discussions.
${ }^{1}$ J.M. Kikkawa and D.D. Awschalom, Phys. Rev. Lett. 80, 4313 (1998).

${ }^{2}$ Y. Ohno et al., Phys. Rev. Lett. 83, 4196 (1999); Y. Ohno et al., Physica E (Amsterdam) 6, 817 (2000).

${ }^{3}$ T. Fujisawa, Y. Tokura, and Y. Hirayama, Phys. Rev. B 63, R81 304 (2001).

${ }^{4}$ D. Loss and D.P. DiVincenzo, Phys. Rev. A 57, 120 (1998).

${ }^{5}$ G. Burkard, D. Loss, and D.P. DiVincenzo, Phys. Rev. B 59, 2070 (1999).

${ }^{6}$ R.C. Ashoori, Nature (London) 379, 413 (1996).

${ }^{7}$ S. Tarucha et al., Phys. Rev. Lett. 77, 3613 (1996).

${ }^{8}$ L.P. Kouwenhoven et al., in Mesoscopic Electron Transport, NATO Advanced Studies Institute Series B: Physics, edited by L.L. Sohn, L.P. Kouwenhoven, and G. Schoen (Kluwer, Dordrecht, 1997).

${ }^{9}$ A.V. Khaetskii and Y.V. Nazarov, Physica E (Amsterdam) 6, 470 (2000).

${ }^{10}$ A.V. Khaetskii and Y.V. Nazarov, Phys. Rev. B 61, 12639 (2000).

${ }^{11}$ D.M. Frenkel, Phys. Rev. B 43, 14228 (1991).

${ }^{12}$ D. Pines, J. Bardeen, and C.P. Slichter, Phys. Rev. 106, 489 (1957).

${ }^{13}$ D. Paget, G. Lampel, and B. Sapoval, Phys. Rev. B 15, 5780 (1977).

${ }^{14}$ D. Paget and V. L. Berkovits, in Optical Orientation, edited by
F. Meier and B.P. Zakharchenya (North-Holland, Amsterdam, 1984).

${ }^{15}$ M. Dobers et al., Phys. Rev. Lett. 61, 1650 (1988).

${ }^{16}$ A. Berg, M. Dobers, R.R. Gerhardts, and K. v. Klitzing, Phys. Rev. Lett. 64, 2563 (1990).

${ }^{17}$ I.D. Vagner, T. Maniv, and E. Ehrenfreund, Solid State Commun. 44, 635 (1982); I.D. Vagner and T. Maniv, Phys. Rev. Lett. 61, 1400 (1988); V.I. Fal'ko, S.V. Meshkov, and I.D. Vagner, J. Phys.: Condens. Matter 3, 5079 (1991); J.H. Kim, I.D. Vagner, and L. Xing, Phys. Rev. B 49, 16777 (1994); I.D. Vagner, Y.A. Bychkov, A.M. Dyugaev, and T. Maniv, Phys. Scr. T66, 158 (1996).

${ }^{18}$ S.E. Barrett et al., Phys. Rev. Lett. 74, 5112 (1995).

${ }^{19}$ R. Tycko et al., Science 268, 1460 (1995).

${ }^{20}$ S.W. Brown, T.A. Kennedy, and D. Gammon, Solid State Nucl. Magn. Reson. 11, 49 (1998).

${ }^{21}$ D. Pfannkuche, $\mathrm{PhD}$ thesis, Habilitationsscrift, Universität Karlshuhe, 1998.

${ }^{22}$ S. Tarucha et al., Physica E (Amsterdam) 3, 112 (1998).

${ }^{23}$ W.G. van der Wiel et al., Physica B 256-258, 173 (1998).

${ }^{24}$ L.P. Kouwenhoven et al., Science 278, 1788 (1997).

${ }^{25} \mathrm{~S}$. Tarucha et al., Physica E (Amsterdam) 3, 112 (1998).

${ }^{26}$ F. Bolton, Phys. Rev. B 54, 4780 (1996). 